\title{
Multi-Party Computation from any Linear Secret Sharing Scheme Unconditionally Secure against Adaptive Adversary: The Zero-Error Case
}

\author{
Ventzislav Nikov ${ }^{1}$, Svetla Nikova ${ }^{2 \star}$, and Bart Preneel $^{2}$ \\ 1 Department of Mathematics and Computing Science, \\ Eindhoven University of Technology \\ P.O. Box 513, 5600 MB, Eindhoven, the Netherlands \\ v.nikov@tue.nl \\ 2 Department Electrical Engineering, ESAT/COSIC, \\ Katholieke Universiteit Leuven, Kasteelpark Arenberg 10, \\ B-3001 Heverlee-Leuven, Belgium \\ svetla.nikova, bart.preneel@esat.kuleuven.ac.be
}

\begin{abstract}
We consider a generalized adaptive and active adversary model for unconditionally secure Multi-Party Computation (MPC) in the zero error case.

Cramer et al. proposed a generic approach to build a multiplicative Monotone Span Programs (MSP) - the special property of a Linear Secret Sharing Schemes (LSSS) that is needed to perform a multiplication of shared values. They give an efficient generic construction to build verifiability into every LSSS and to obtain from any LSSS a multiplicative LSSS for the same access structure. But the multiplicative property guarantees security against passive adversary only. For an active adversary a strong multiplicative property is required. Unfortunately there is no known efficient construction to obtain a strongly multiplicative LSSS yet.

Recently Nikov et al. have expanded the construction of Cramer et al. using a different approach. Multiplying two different MSP $M_{1}$ and $M_{2}$ computing the access structures $\Gamma_{1}$ and $\Gamma_{2}$ a new MSP $M$ called "resulting" is obtained. $M$ computes a new access structure $\Gamma \subset \Gamma_{1} \quad\left(\right.$ or $\left.\Gamma_{2}\right)$. The goal of this construction is to enable the investigation of how the properties that $\Gamma$ should fulfil are linked to the initial access structures $\Gamma_{1}$ and $\Gamma_{2}$. It is proved that $\Gamma_{2}$ should be a dual access structure of $\Gamma_{1}$ in order to have a multiplicative resulting MSP. But there are still not known requirements for initial access structures in order to obtain strongly multiplicative resulting MSP. Nikov et al. proved that to have unconditionally secure MPC the following minimal conditions for the resulting access structure should be satisfied $\left(\Gamma_{A} \uplus \Gamma_{A}\right)^{\perp} \subseteq \Gamma$.

In this paper we assume that the resulting MSP could be constructed such that the corresponding access structure $\Gamma$ satisfies the required
\end{abstract}

\footnotetext{
* The author was partially supported by IWT and Concerted Research Action GOAMEFISTO-666 of the Flemish Government
} 
properties. Our goal is to study the requirements that $\Gamma$ should fulfil in order to have an MPC unconditionally secure against adaptive and active adversary in the zero error case. First, we prove that $\Gamma$ could satisfy weaker conditions than those in Nikov et al., namely $\Gamma_{A}^{\perp} \subseteq \Gamma$. Second, we propose a commitment "degree reduction" protocol which allows the players to "reduce" one access structure, e.g. $\Gamma$, to another access structure $\Gamma_{3}$. This reduction protocol appears to be a generalization of the reduction protocol of Cramer et al. in the sense that we can choose to reduce $\Gamma$ to the initial access structures $\Gamma_{1}$ or $\Gamma_{2}$, or to a new one $\Gamma_{3}$. This protocol is also more efficient, since it requires less Verifiable Secret Sharing Schemes to be used.

Keywords: general secure multi-party computation, verifiable secret sharing, linear secret sharing, monotone span programs, general adversaries, information theoretic security.

\section{Introduction}

Secure multi-party computation (MPC) can be defined as follows: $n$ players compute an agreed function of their inputs in a "secure" way, where "secure" means guaranteeing the correctness of the output as well as the privacy of the players' inputs, even when some players cheat. A key tool for secure MPC, is the verifiable secret sharing (VSS) [6, 1]. In VSS a dealer distributes a secret value among the players, where the dealer and/or some of the players may be cheating. It is guaranteed that if the dealer is honest, then the cheaters obtain no information about the secret, and all honest players will later be able to reconstruct it, without the help of the dealer. Even if the dealer cheats, a unique value will be determined and is reconstructible without the cheaters' help.

In [18] Shamir introduced the concept of secret sharing as a tool to protect a secret simultaneously from exposure and from being lost. It allows a so called dealer to share the secret among a set of entities, usually called players, in such a way that only certain specified subsets of the players are able to reconstruct the secret while smaller subsets have no information about it. The groups who are allowed to reconstruct the secret are called qualified, and the groups who should not be able to obtain any information about the secret are called forbidden. The collection of all qualified groups is denoted by $\Gamma$, and the collection of all forbidden groups is denoted by $\Delta$. The tuple $(\Gamma, \Delta)$ is called an access structure if $\Gamma \cap \Delta=\emptyset$. Denote by $P=\left\{P_{1}, \ldots, P_{n}\right\}$ the set of participants in the scheme and by $\mathcal{P}(P)$ the set of all subsets of $P$. If $\Gamma \cup \Delta=\mathcal{P}(P)$, i.e., $\Gamma=\Delta^{c}$ is the complement 
of $\Delta$, then $(\Gamma, \Delta)$ is complete and it is denoted simply by $\Gamma$. When $\Gamma$ is complete the SSS is called perfect.

Usually the cheating is represented as an adversary who may corrupt some subset of the players. One can distinguish between passive and active corruption, see Fehr and Maurer, [8] for recent results. Passive corruption means that the adversary obtains the complete information held by the corrupt players, but the players execute the protocol correctly. Active corruption means that the adversary takes full control of the corrupt players. Active corruption is strictly stronger than passive corruption. The adversary is characterized by a privacy structure $\Delta$ and an adversary structure $\Delta_{A} \subseteq \Delta$. Denote the complement $\Gamma_{A}=\Delta_{A}^{c}$ and call its dual access structure $\Gamma_{A}^{\perp}$ the honest (or good) players structure. Both passive and active adversaries may be static, meaning that the set of corrupt players is chosen once and for all before the protocol starts, or adaptive meaning that the adversary can at any time during the protocol choose to corrupt a new player based on all the information he has at the time, as long as the total set is in $\Delta_{A}$.

Most proposed Secret Sharing Schemes (SSS) are linear, but the concept of a Linear Secret Sharing Scheme (LSSS) was first considered in its full generality by Karchmer and Wigderson in [13], who introduced the equivalent notion of Monotone Span Program (MSP), which we describe later. Each linear SSS can be viewed as derived from a monotone span program $\mathcal{M}$ computing its access structure. On the other hand, each monotone span program gives rise to an LSSS. Hence, one can identify an LSSS with its underlying monotone span program. Such an MSP always exists, because MSPs can compute any monotone function. Since an LSSS neither guarantees reconstructability when some shares are incorrect, nor verifiability of a shared value the stronger primitive - Verifiable Secret Sharing has been introduced.

We will consider any complete general monotone access structure $\Gamma$, which describes subsets of participants that are qualified to recover the secret $s \in \mathbb{F}$ ( $\mathbb{F}$ here is a finite field) in the set of possible secret values, as long as it admits a linear secret sharing scheme. We will consider also the standard synchronous model with a broadcast channel.

\subsection{Related Work}

This subsection contains some basic definitions, notations and results. For an arbitrary matrix $M$ over $\mathbb{F}$, with $m$ rows labelled by $1, \ldots, m$ let $M_{A}$ denote the matrix obtained by keeping only those rows $i$ with $i \in A$, where $A$ is an arbitrary non-empty subset of $\{1, \ldots, m\}$. If $\{i\}=A$ we 
write $M_{i}$. Let $M_{A}^{T}$ denote the transpose of $M_{A}$, and let $\operatorname{Im}\left(M_{A}^{T}\right)$ denote the $\mathbb{F}$-linear span of the rows of $M_{A}$. We use $\operatorname{Ker}\left(M_{A}\right)$ to denote the kernel of $M_{A}$, i.e., all linear combinations of the columns of $M_{A}$, leading to 0 .

Let $v=\left(v_{1}, \ldots, v_{t_{1}}\right) \in \mathbb{F}^{t_{1}}$ and $w=\left(w_{1}, \ldots, w_{t_{2}}\right) \in \mathbb{F}^{t_{2}}$ be two vectors. The tensor vector product $v \otimes w$ is defined as a vector in $\mathbb{F}^{t_{1} t_{2}}$ such that the $j$-coordinate in $v$ (denoted by $v_{j}$ ) is replaced by $v_{j} w$, i.e., $v \otimes w=$ $\left(v_{1} w, \ldots, v_{t_{1}} w\right) \in \mathbb{F}^{t_{1} t_{2}}$. The Kronecker product of matrices is defined as tensor vector multiplication of each row from the first matrix to each row from the second matrix.

Definition 1. [5] The dual $\Gamma^{\perp}$ of a monotone access structure $\Gamma$ defined on $P$ is the collection of sets $A \subseteq P$ such that $A^{c} \notin \Gamma$.

The following operation (called element-wise union) for monotone decreasing (increasing) sets was introduced in $[15,8]$.

Definition 2. For monotone decreasing sets $\Delta_{1}, \Delta_{2}$ and for monotone increasing sets $\Gamma_{1}, \Gamma_{2}$, all defined for the same set of participants, the element-wise union operation $*$ is defined by:

$$
\begin{aligned}
\Delta_{1} * \Delta_{2} & =\left\{A_{1} \cup A_{2} ; A_{1} \in \Delta_{1}, A_{2} \in \Delta_{2}\right\}, \\
\text { resp. } \quad \Gamma_{1} * \Gamma_{2} & =\left\{A_{1} \cup A_{2} ; A_{1} \notin \Gamma_{1}, A_{2} \notin \Gamma_{2}\right\}^{c} .
\end{aligned}
$$

Throughout the paper we will consider presence of adaptive adversary. Let $Q^{2}$, resp. $Q^{3}$ be the conditions on an adversary structure that no two, resp. no three of the sets in the structure cover the full players set $P$. The adversary that we tolerate is at least a $Q^{2}$ (resp. $Q^{3}$ ) adversary in the passive (resp. active) scenario (see $[12,4]$ ). Since the condition $Q^{2}$ is equivalent to $\Delta_{A} \cap \Gamma_{A}^{\perp}=\emptyset$ (i.e., $\Gamma_{A}^{\perp} \subseteq \Gamma_{A}$ ), the honest players structure has no intersection with the adversary structure.

Recently Maurer [14] proved that general perfect information-theoretically secure MPC secure against a $\left(\Delta_{1}, \Delta_{A}\right)$-adversary is possible if and only if $P \notin \Delta_{1} \uplus \Delta_{1} \uplus \Delta_{A}$ or equivalently, if and only if $\Gamma_{A}^{\perp} \subseteq \Gamma_{1} \uplus \Gamma_{1}$. Maurer consider the case, when the secrets are shared using only one MSP. Notice that thanks to the local computation model for MPC the interaction between players is reduced, and in this way we may think of the MPC as a kind of VSS.

A recent result, which gives necessary and sufficient conditions for the existence of information-theoretically secure VSS has been presented by Fehr and Maurer in [8]. They prove that the robustness conditions for 
VSS are fulfilled if and only if $P \notin \Delta \uplus \Delta_{A} \uplus \Delta_{A}$ or equivalently, if and only if $\left(\Gamma_{A} \uplus \Gamma_{A}\right)^{\perp} \subseteq \Gamma$.

As mentioned earlier, MSPs are essentially equivalent to LSSS's (see e.g. [13]). It turns out to be convenient to describe our protocols in terms of MSPs as we will do for the rest of the paper. A formal definition for an MSP follows.

Definition 3. [3, 4] A Monotone Span Program (MSP) $\mathcal{M}$ is a quadruple $(\mathbb{F}, M, \varepsilon, \psi)$, where $\mathbb{F}$ is a finite field, $M$ is a matrix (with $m$ rows and $d \leq m$ columns) over $\mathbb{F}, \psi:\{1, \ldots, m\} \rightarrow\{1, \ldots, n\}$ is a surjective function and $\varepsilon$ is a fixed vector, called target vector, e.g. column vector $(1,0, \ldots, 0) \in \mathbb{F}^{d}$. The size of $\mathcal{M}$ is the number $m$ of rows.

As $\psi$ labels each row with a number from $[1, \ldots, m]$ corresponding to a fixed player, we can think of each player as being the "owner" of one or more rows. For every player we consider a function $\varphi$ which gives the set of rows owned by the player, i.e., $\varphi$ is (in some sense) inverse of $\psi$.

An MSP is said to compute a (complete) access structure $\Gamma$ when $\varepsilon \in$ $\operatorname{Im}\left(M_{\varphi(G)}^{T}\right)$ if and only if $G$ is a member of $\Gamma$. Hence, the players can reconstruct the secret precisely if the rows they own contain in their linear span the target vector of $\mathcal{M}$, and otherwise they get no information about the secret, i.e., there exists a so called recombination vector $\mathbf{r}$ such that $\left\langle\mathbf{r}, M_{\varphi(G)}(s, \rho)\right\rangle=s$ and $M_{\varphi(G)}^{T} \mathbf{r}=\varepsilon$ for any secret $s$ and any $\rho$. It is well known that the vector $\varepsilon \notin \operatorname{Im}\left(M_{N}^{T}\right)$ if and only if there exists a $\mathbf{k} \in \mathbb{F}^{d}$ such that $M_{N} \mathbf{k}=0$ and $\mathbf{k}_{1}=1$.

The main goal of our paper is to study the properties of a construction which builds MPCs from any LSSS. It is well known that because of the linearity the LSSS provides it is easy to add secrets securely. Therefore to achieve general MPC, it suffices to implement multiplication of shared secrets. That is, we need a protocol where each player initially holds shared secrets $s$ and $s^{\prime}$, and ends up holding a share of the product $s s^{\prime}$. Several such protocols are known for the threshold case $[1,2,10,11]$ and for general access structure $[3,4,17]$.

We follow the approach proposed by Cramer et al. in $[3,4]$ to build an MPC from any LSSS, provided that the LSSS is what is called (strongly) multiplicative. Loosely speaking, an LSSS is (strongly) multiplicative if each player $P_{i}$ can compute from his shares (of secrets $s$ and $s^{\prime}$ ) a value $c_{i}$, such that the product $s s^{\prime}$ can be obtained using all values (only values from honest players).

In a recent paper by Nikov et al. [17] the $\diamond$ construction for multiplying two MSPs has been proposed. Let $\Gamma_{1}$ and $\Gamma_{2}$ be access structures, 
computed by MSPs $\mathcal{M}_{1}=\left(\mathbb{F}, M_{1}, \varepsilon_{1}, \psi_{1}\right)$ and $\mathcal{M}_{2}=\left(\mathbb{F}, M_{2}, \varepsilon_{2}, \psi_{2}\right)$. Let also $M_{1}$ be an $m_{1} \times d_{1}$ matrix, $M_{2}$ be an $m_{2} \times d_{2}$ matrix and $\varphi_{1}, \varphi_{2}$ be the "inverse" functions of $\psi_{1}$ and $\psi_{2}$. Consider the vector $x$. The coordinates in $x$, which belong to the player $t$ are collected in a sub-vector $x_{t}$ or $x=\left(\bar{x}_{1}, \ldots, \bar{x}_{n}\right)$. First the operation $\diamond$ for vectors is defined as follows:

$$
x \diamond y=\left(\bar{x}_{1} \otimes \bar{y}_{1}, \ldots, \bar{x}_{n} \otimes \bar{y}_{n}\right) .
$$

Denote by $\left(M_{1}\right)_{\mathbf{t}}$ the matrix formed by rows of $M_{1}$ owned by the player $t$ and correspondingly by $\left(M_{2}\right)_{\mathbf{t}}$ the matrix formed by rows of $M_{2}$ owned by the same player. Hence $M_{1}$ can be presented as a concatenation of the matrices $\left(M_{1}\right)_{\mathbf{t}}$ for $t=1, \ldots, n$. Then the operation $\diamond$ for matrices is defined as the concatenation of matrices $\left(M_{1}\right)_{\mathbf{t}} \otimes\left(M_{2}\right)_{\mathbf{t}}$ for $t=1, \ldots, n$, i.e.,

$$
M=M_{1} \diamond M_{2}=\left(\begin{array}{c}
\left(M_{1}\right)_{\mathbf{1}} \otimes\left(M_{2}\right)_{\mathbf{1}} \\
\cdots \\
\left(M_{1}\right)_{\mathbf{n}} \otimes\left(M_{2}\right)_{\mathbf{n}}
\end{array}\right) .
$$

Finally, the operation $\diamond$ for two MSP could be defined as:

Definition 4. [17] Define $M S P \mathcal{M}$ to be $\left(\mathbb{F}, M=M_{1} \diamond M_{2}, \varepsilon=\varepsilon_{1} \diamond \varepsilon_{2}, \psi\right)$, where $\psi(i, j)=r$ if and only if $\psi_{1}(i)=\psi_{2}(j)=r$ and the size of $\mathcal{M}$ is $m=\sum_{i}\left|\varphi_{1}(i)\right|\left|\varphi_{2}(i)\right|=\sum_{i}|\varphi(i)|$. Given two MSPs $\mathcal{M}_{1}$ and $\mathcal{M}_{2}$, the $M S P \mathcal{M}$ is called their multiplicative resulting MSP and denoted by $\mathcal{M}=\mathcal{M}_{1} \diamond \mathcal{M}_{2}$ if there exists an $m$-vector $\mathbf{r}$ called a recombination vector, such that for any two secrets $s^{\prime}$ and $s^{\prime \prime}$ and any $\rho^{\prime}$ and $\rho^{\prime \prime}$, it holds that

$$
s^{\prime} s^{\prime \prime}=\left\langle\mathbf{r}, M_{1}\left(s^{\prime}, \rho^{\prime}\right) \diamond M_{2}\left(s^{\prime \prime}, \rho^{\prime \prime}\right)\right\rangle=\left\langle\mathbf{r}, M\left(\left(s^{\prime}, \rho^{\prime}\right) \otimes\left(s^{\prime \prime}, \rho^{\prime \prime}\right)\right)\right\rangle .
$$

The MSP $\mathcal{M}$ is called their strongly multiplicative resulting MSP if the access structure $\Gamma$ computed by $\mathcal{M}$ is such that for any players' subset $A \in \Gamma, \mathcal{M}_{A}$ is the multiplicative resulting $M S P$ of $\left(\mathcal{M}_{1}\right)_{A}$ and $\left(\mathcal{M}_{2}\right)_{A}$.

The last definition means that one can construct a strongly multiplicative resulting MSP, computing the product of the secrets shared by MSPs $\mathcal{M}_{1}$ and $\mathcal{M}_{2}$, with some access structure $\Gamma$. The difference between the multiplicative resulting MSP and the strongly multiplicative resulting MSP is that in the first case $\Gamma=\{P\}$.

It has been proved in [17] that $\Gamma \subseteq \Gamma_{1} \uplus \Gamma_{2}$. In the model of MPC proposed in [17] the secrets are shared using VSS and two MSP $\mathcal{M}_{1}$ and $\mathcal{M}_{2}$. Hence the adaptive adversary has two privacy structures $\Delta_{1}, \Delta_{2}$ and one adversary structure $\Delta_{A} \subseteq \Delta_{1}, \Delta_{A} \subseteq \Delta_{2}$. Such an adversary is denoted by $\left(\Delta_{1}, \Delta_{2}, \Delta_{A}\right)$-adversary. 
In the computational model for MPC the authors in [17] propose the so called "algebraic simplification for multiplication" protocol which uses homomorphic commitments in the strongly multiplicative case of general MPC. In fact, the "algebraic simplification for multiplication" protocol allows the players to "reduce" one access structure $\Gamma$ to another access structure $\Gamma_{3}$, provided that the VSS conditions for $\Gamma_{3}$ hold. As it is proved in [17] to build a MPC protocol secure against an adaptive adversary in the computational model it is sufficient the MSPs $\mathcal{M}_{1}, \mathcal{M}_{2}, \mathcal{M}_{3}$ to satisfy the VSS conditions, i.e., $\Gamma_{A}^{\perp} \subseteq \Gamma_{i}$ for $i=1,2,3 ; \mathcal{M}$ to be resulting MSP of $\mathcal{M}_{1}$ and $\mathcal{M}_{2}$, i.e., $\Gamma \subseteq \Gamma_{1} \uplus \Gamma_{2}$ and $\Gamma$ to satisfy the strong multiplicative property, i.e., $\Gamma_{A}^{\perp} \subseteq \Gamma$. On the other hand the lack of "algebraic simplification for multiplication" protocol in the information-theoretic scenario impose stronger conditions for the strongly multiplicative case of general MPC. It is proved in [17] that it is sufficient for the MSPs $\mathcal{M}_{1}$ and $\mathcal{M}_{2}$ to satisfy the VSS conditions from [8], i.e., $\left(\Gamma_{A} \uplus \Gamma_{A}\right)^{\perp} \subseteq \Gamma_{i}$ for $i=1,2$; $\mathcal{M}$ to be resulting MSP of $\mathcal{M}_{1}$ and $\mathcal{M}_{2}$, i.e., $\Gamma \subseteq \Gamma_{1} \uplus \Gamma_{2}$ and $\Gamma$ to satisfy the following property,

$$
\left(\Gamma_{A} \uplus \Gamma_{A}\right)^{\perp} \subseteq \Gamma
$$

\subsection{Results of This Paper}

The condition (1) is sufficient to multiply securely two secrets, but it is insufficient to perform general MPC, since with each multiplication the access structure $\Gamma$ becomes "smaller" and "smaller". Hence besides multiplying securely we need a "degree reduction" protocol to "reduce" the access structure $\Gamma$ to another access structure e.g. $\Gamma_{3}$. The solution that we propose is parallel to the one in the threshold case, where after multiplication we have threshold $2 t$ and reduce it to threshold $t$ as Ben-Or et al. show in [1].

In this paper we build an information-theoretically secure simplification protocol for multiplication, which is an important step in order to be achieved general secure MPC. The main hurdle to overcome in the "degree reduction" protocol is the additional check which ensures the commitment to the re-shared shares. The clue in this additional check is the change of the basis (see Section 3.3).

Our main result follows:

Theorem 1. Suppose that for the $M S P s \mathcal{M}_{1}$ and $\mathcal{M}_{2}$ there exist MSPs $\mathcal{M}_{3}$ and $\mathcal{M}_{4}$ such that $\mathcal{M}_{1} \diamond \mathcal{M}_{2}=\mathcal{M}=\mathcal{M}_{3} \diamond \mathcal{M}_{4}$. Then the sufficient condition for existence of general perfect information-theoretically secure 
MPC secure against $\left(\Delta_{1}, \Delta_{2}, \Delta_{A}\right)$-adversary is

$$
\Gamma_{A}^{\perp} \subseteq \Gamma \subseteq \Gamma_{1} \uplus \Gamma_{2}, \quad\left(\Gamma_{A} \uplus \Gamma_{A}\right)^{\perp} \subseteq \Gamma_{i} \quad \text { for } i=1,2,3,
$$

where $\Gamma$ is the access structure computed by the strongly multiplicative resulting $M S P \mathcal{M}$ from $M S P s \mathcal{M}_{1}$ and $\mathcal{M}_{2}$ and/or from $M S P s \mathcal{M}_{3}$ and $\mathcal{M}_{4}$.

We will call the access structure $\Gamma_{3}$ (the MSP $\mathcal{M}_{3}$, resp.) "reduced". It is easy to see that such MSPs $\mathcal{M}_{3}$ and $\mathcal{M}_{4}$ always exist, e.g. $\mathcal{M}_{1}=\mathcal{M}_{3}$ and $\mathcal{M}_{2}=\mathcal{M}_{4}$. In the threshold case there exist several pairs of MSPs that satisfy the assumption of Theorem 1 .

Note also that the Maurer's [14] necessary and sufficient condition $P \notin$ $\Delta_{1} \uplus \Delta_{1} \uplus \Delta_{A}$ is satisfied (in case $\Gamma_{1}=\Gamma_{2}$ ), on the other hand this conditions does not guarantee that $\Gamma_{A}^{\perp} \subseteq \Gamma$, when $\Gamma \neq \Gamma_{1} \uplus \Gamma_{2}$, i.e., $\Gamma \subset \Gamma_{1} \uplus \Gamma_{2}$.

The picture in the general access structure appears to be analogous to this in the threshold case $[7,9]$. Remarkably the conditions in the informationtheoretic settings are "similar" to the conditions in the cryptographic settings (see the result of Nikov et al. for the computational model). Note that it is not required anymore $\Gamma$ to satisfy the VSS conditions.

If we compare with the protocol in [4] we can see that now the player who re-shares his share do not need to commit to every single entry in the used vector. Hence the number of the used VSS is reduced. Also note that this protocol does not depend on the model considered here (Nikov et al.), it could be applied also for the model of Cramer et al.

The paper is organized as follows: In Section 2 the information-theoretically secure VSS, randomization and re-sharing protocols are presented. In Section 3 we introduce some terminology and concepts, we state the results and explain the role they play in comparison with earlier results.

\section{Background}

\subsection{VSS - Share Phase}

Let the dealer $\mathcal{D}$ shares the secret $s$ to the players $P_{i}$ using the VSS protocol, as described by Cramer et al. in [4], and let $\mathcal{M}$ be an MSP with matrix $M(m \times d)$.

1. The Dealer $\mathcal{D}$ chooses a symmetric $d \times d$ matrix $R$ subject to $s$ (the secret) in its upper left corner. 
2. The Dealer $\mathcal{D}$ gives to the participant $P_{i}$ shares $v_{\varphi(i)}=M_{\varphi(i)} R\left(v_{\varphi(i)}\right.$ is $|\varphi(i)| \times d$ matrix), where the "true part" (which will be used in the reconstruction) of the shares is $v_{\varphi(i)} \varepsilon$.

3. The players $P_{i}$ and $P_{j}$ perform a pairwise-check as follows:

$$
M_{\varphi(j)} v_{\varphi(i)}^{T}=M_{\varphi(j)} R M_{\varphi(i)}^{T}=v_{\varphi(j)} M_{\varphi(i)}^{T} .
$$

\subsection{VSS - Reconstruction Phase}

For any group of players $G \in \Gamma$ there exists a recombination vector $\lambda_{\varphi(G)}$, such that they can reconstruct together the secret $s$ as follows:

$$
\left(v_{\varphi(G)} \varepsilon\right) \lambda_{\varphi(G)}^{T}=\left\langle\lambda_{\varphi(G)}, v_{\varphi(G)} \varepsilon\right\rangle=\sum_{i \in G} \lambda_{\varphi(i)}\left(v_{\varphi(i)} \varepsilon\right)=s .
$$

\subsection{Information-Theoretic Homomorphic Commitments and Re-Share Phase}

In the re-share phase each player $P_{i}$ plays the role of the dealer sharing the true part of his shares among the participants using VSS with the same MSP $\mathcal{M}$.

1. Any player $P_{i}$ re-shares his true part of the share $v_{\varphi(i)} \varepsilon$, i.e., for any $i_{1} \in \varphi(i)$ he chooses a symmetric $d \times d$ matrix $R^{\left(i_{1}\right)}$ such that its first row (column) is $v_{i_{1}}$ and the value in its upper left corner is $v_{i_{1}} \varepsilon$.

2. $P_{i}$ sends to $P_{j}$ temporary shares $y_{i_{1}, \varphi(j)}=M_{\varphi(j)} R^{\left(i_{1}\right)}$, whose true part is $y_{i_{1}, \varphi(j)} \varepsilon$.

3. The players $P_{k}$ and $P_{j}$ perform the usual commitment verification (VSS pairwise-check):

$$
M_{\varphi(j)} y_{i_{1}, \varphi(k)}^{T}=M_{\varphi(j)} R^{\left(i_{1}\right)} M_{\varphi(k)}^{T}=y_{i_{1}, \varphi(j)} M_{\varphi(k)}^{T} .
$$

4. In addition $P_{j}$ checks his true part of the share

$$
y_{i_{1}, \varphi(j)} \varepsilon=M_{\varphi(j)} R^{\left(i_{1}\right)} \varepsilon=M_{\varphi(j)} v_{i_{1}}^{T}=v_{\varphi(j)} M_{i_{1}}^{T} .
$$

The last equality is the pair-wise check in VSS (step 3 in the Share phase). Note that this additional check ensures that the player $P_{i}$ really re-shares his share, i.e., he is honest.

5. As usual for any group of players $\widetilde{G} \in \Gamma$ there exists a recombination vector $\widetilde{\lambda}_{\varphi(\widetilde{G})}$ such that they can together reconstruct the true part of the initial share $-v_{i_{1}} \varepsilon$.

$$
\left(y_{i_{1}, \varphi(\widetilde{G})} \varepsilon\right) \widetilde{\lambda}_{\varphi(\widetilde{G})}^{T}=\left\langle\widetilde{\lambda}_{\varphi(\widetilde{G})}, y_{i_{1}, \varphi(\widetilde{G})} \varepsilon\right\rangle=\sum_{j \in \widetilde{G}} \widetilde{\lambda}_{\varphi(j)}\left(y_{i_{1}, \varphi(j)} \varepsilon\right)=v_{i_{1}} \varepsilon
$$


6. Denote the list of good players by $\mathcal{L} \in \Gamma$. Then $P_{j}$, using the corresponding recombination vector $\lambda_{\varphi(\mathcal{L})}$, computes

$$
z_{\varphi(j)}=\sum_{i \in \mathcal{L}} \lambda_{\varphi(i)} y_{\varphi(i), \varphi(j)} .
$$

The new shares (of the same secret $s$ ) are $z_{\varphi(j)}$ and they satisfy all the necessary properties as follows:

- The pair-wise check holds:

$$
\begin{aligned}
M_{\varphi(k)} z_{\varphi(j)}^{T} & =\sum_{i \in \mathcal{L}} \lambda_{\varphi(i)} M_{\varphi(k)} y_{\varphi(i), \varphi(j)}^{T} \\
& =\left(\sum_{i \in \mathcal{L}} \lambda_{\varphi(i)} y_{\varphi(i), \varphi(k)}\right) M_{\varphi(j)}^{T}=z_{\varphi(k)} M_{\varphi(j)}^{T} .
\end{aligned}
$$

- The players in any group $\widetilde{G} \in \Gamma$ can reconstruct the secret $s$ together.

$$
\begin{aligned}
\left(z_{\varphi(\widetilde{G})} \varepsilon\right) \widetilde{\lambda}_{\varphi(\widetilde{G})}^{T} & =\left\langle\widetilde{\lambda}_{\varphi(\widetilde{G})}, z_{\varphi(\widetilde{G})} \varepsilon\right\rangle=\sum_{j \in \widetilde{G}} \widetilde{\lambda}_{\varphi(j)}\left(z_{\varphi(j)} \varepsilon\right) \\
& =\sum_{j \in \widetilde{G}} \widetilde{\lambda}_{\varphi(j)}\left(\sum_{i \in \mathcal{L}} \lambda_{\varphi(i)}\left(y_{\varphi(i), \varphi(j)} \varepsilon\right)\right) \\
& =\sum_{i \in \mathcal{L}} \lambda_{\varphi(i)}\left(\sum_{j \in \widetilde{G}} \widetilde{\lambda}_{\varphi(j)}\left(y_{\varphi(i), \varphi(j)} \varepsilon\right)\right)=\sum_{i \in \mathcal{L}} \lambda_{\varphi(i)}\left(v_{\varphi(i)} \varepsilon\right)=s .
\end{aligned}
$$

\subsection{The Randomization Phase}

We can use the Renewal phase from [16] as a randomization protocol.

\section{Reduction Protocol}

\subsection{The Set-up}

Let $\Gamma_{1}$ and $\Gamma_{2}$ be access structures, computed by MSPs $\mathcal{M}_{1}=\left(\mathbb{F}, M_{1}, \varepsilon_{1}, \psi_{1}\right)$ and $\mathcal{M}_{2}=\left(\mathbb{F}, M_{2}, \varepsilon_{2}, \psi_{2}\right)$, respectively. Let also $M_{1}$ be $m_{1} \times d_{1}$ matrix, $M_{2}$ be $m_{2} \times d_{2}$ matrix and $\varphi_{1}, \varphi_{2}$ be the "inverse" functions of $\psi_{1}$ and $\psi_{2}$.

Let $\mathcal{M}=\mathcal{M}_{1} \diamond \mathcal{M}_{2}$ be the multiplicative resulting $\mathrm{MSP}$, i.e., $\mathcal{M}=$ $\left(\mathbb{F}, M=M_{1} \diamond M_{2}, \varepsilon=\varepsilon_{1} \diamond \varepsilon_{2}, \psi\right)$, where $\psi(i, j)=r$ if and only if $\psi_{1}(i)=$ $\psi_{2}(j)=r$. Hence $M$ is $m \times d_{1} d_{2}$ matrix, where $m=\sum_{i}\left|\varphi_{1}(i)\right|\left|\varphi_{2}(i)\right|=$ $\sum_{i}|\varphi(i)|$. Let us consider the access structure $\Gamma$ computed by the MSP $\mathcal{M}$. 
Let the first secret $s_{1}$ is shared using VSS by MSP $\mathcal{M}_{1}$ with symmetric $d_{1} \times d_{1}$ matrix $R^{(1)}$, i.e., $v_{\varphi_{1}(i)}=\left(M_{1}\right)_{\varphi_{1}(i)} R^{(1)}$ be the shares of $P_{i}\left(v_{\varphi_{1}(i)}\right.$ is $\left|\varphi_{1}(i)\right| \times d_{1}$ matrix). The "true part" of the shares are the first coordinates of each share, i.e., $v_{\varphi_{1}(i)} \varepsilon_{1}$.

Analogously, let the second secret $s_{2}$ is shared by MSP $\mathcal{M}_{2}$ with symmetric $d_{2} \times d_{2}$ matrix $R^{(2)}$, i.e., $w_{\varphi_{2}(i)}=\left(M_{2}\right)_{\varphi_{2}(i)} R^{(2)}$ be the shares of $P_{i}$. $\left(w_{\varphi_{2}(i)}\right.$ is $\left|\varphi_{2}(i)\right| \times d_{2}$ matrix). The "true part" of the shares are the first coordinates of each share, i.e., $w_{\varphi_{2}(i)} \varepsilon_{2}$.

\subsection{Local Computation Phase}

Denote by $R=R^{(1)} \otimes R^{(2)}$ a $d_{1} d_{2} \times d_{1} d_{2}$ symmetric matrix. Note that the value in the upper left corner of $R$ is the product $s_{1} s_{2}$. Let us choose the indices $i_{1} \in \varphi_{1}(i), i_{2} \in \varphi_{2}(i), j_{1} \in \varphi_{1}(j)$ and $j_{2} \in \varphi_{2}(j)$.

If the player $P_{i}$ locally computes $\otimes$ product of his shares he obtains his new shares $v_{\varphi_{1}(i)} \otimes w_{\varphi_{2}(i)}$ (which are an $|\varphi(i)| \times d_{1} d_{2}$ matrix).

This shares correspond to an MSP $\mathcal{M}$ and the random matrix $R$ as defined above, i.e., $\left(\left(M_{1}\right)_{i_{1}} \otimes\left(M_{2}\right)_{i_{2}}\right) R=v_{i_{1}} \otimes w_{i_{2}}$.

The pair-wise check for the new shares also holds:

$$
\begin{aligned}
\left(\left(M_{1}\right)_{i_{1}} \otimes\left(M_{2}\right)_{i_{2}}\right)\left(v_{j_{1}} \otimes w_{j_{2}}\right)^{T} & =\left(\left(M_{1}\right)_{i_{1}} v_{j_{1}}^{T}\right)\left(\left(M_{2}\right)_{i_{2}} w_{j_{2}}^{T}\right)= \\
\left(v_{i_{1}}\left(M_{1}\right)_{j_{1}}^{T}\right)\left(w_{i_{2}}\left(M_{2}\right)_{j_{2}}^{T}\right) & =\left(v_{i_{1}} \otimes w_{i_{2}}\right)\left(\left(M_{1}\right)_{j_{1}} \otimes\left(M_{2}\right)_{j_{2}}\right)^{T} .
\end{aligned}
$$

Note that the new "true part" of the shares is the product

$$
\left(v_{\varphi_{1}(i)} \otimes w_{\varphi_{2}(i)}\right) \varepsilon=\left(v_{\varphi_{1}(i)} \varepsilon_{1}\right) \otimes\left(w_{\varphi_{2}(i)} \varepsilon_{2}\right) .
$$

In the new MSP $\mathcal{M}$ for any group of players $G \in \Gamma$ there exists a recombination vector $\lambda_{\varphi(G)}$ such that they can reconstruct together the product of the secrets $-s_{1} s_{2}$.

$$
\begin{aligned}
\left(\left(v_{\varphi_{1}(G)} \otimes w_{\varphi_{2}(G)}\right) \varepsilon\right) \lambda_{\varphi(G)}^{T} & =\left\langle\lambda_{\varphi(G)},\left(v_{\varphi_{1}(G)} \otimes w_{\varphi_{2}(G)}\right) \varepsilon\right\rangle \\
& =\sum_{j \in G} \lambda_{\varphi(j)}\left(\left(v_{\varphi_{1}(j)} \otimes w_{\varphi_{2}(j)}\right) \varepsilon\right)=s_{1} s_{2} .
\end{aligned}
$$

\subsection{Decomposition - Change of the Basis}

Let $d_{3}$ and $d_{4}$ are integers such that $d_{1} d_{2}=d_{3} d_{4}$ and, as usual, $\varepsilon_{3} \in \mathbb{F}^{d_{3}}$ be the unit column vector. Denote by $e_{i}=(0, \ldots, 0,1,0, \ldots, 0) \in \mathbb{F}^{d_{4}}$ the unit row vectors, for $i=1, \ldots, d_{4}$. 
It is easy to see that there exist uniquely defined vectors $x_{j_{1}, j_{2}}^{(i)}, \widetilde{x}_{j_{1}, j_{2}}^{(i)} \in \mathbb{F}^{d_{3}}$ for $i=1, \ldots, d_{4}$, such that the following equalities hold

$$
v_{j_{1}} \otimes w_{j_{2}}=\sum_{i=1}^{d_{4}} x_{j_{1}, j_{2}}^{(i)} \otimes e_{i} ; \quad v_{j_{1}} \otimes w_{j_{2}}=\sum_{i=1}^{d_{4}} e_{i} \otimes \widetilde{x}_{j_{1}, j_{2}}^{(i)} .
$$

Note that $\left(v_{j_{1}} \otimes w_{j_{2}}\right) \varepsilon=x_{j_{1}, j_{2}}^{(1)} \varepsilon_{3}=\widetilde{x}_{j_{1}, j_{2}}^{(1)} \varepsilon_{3}$.

\subsection{Degree Reduction Phase}

Let $\Gamma_{3}$ be an access structure, computed by the MSP $\mathcal{M}_{3}=\left(\mathbb{F}, M_{3}, \varepsilon_{3}, \psi_{3}\right)$. Let also $M_{3}$ be $m_{3} \times d_{3}$ matrix and $\varphi_{3}$ be the "inverse" functions of $\psi_{3}$. Any player $P_{j}$ re-shares the first coordinate of the vector $x_{j_{1}, j_{2}}^{(i)}$, i.e., $x_{j_{1}, j_{2}}^{(i)} \varepsilon_{3}$ for $i=1, \ldots, d_{4}$ using VSS Share protocol. Let us denote the different copies of VSSs by $V S S(i)$. For each VSS the player uses a symmetric $d_{3} \times d_{3}$ matrix $R_{j_{1}, j_{2}}^{(i)}$, such that its first row (column) is $x_{j_{1}, j_{2}}^{(i)}$. So, the player $P_{k}$ receives from $P_{j}$ the following temporary shares:

$$
y_{j_{1}, j_{2}, \varphi_{3}(k)}^{(i)}=\left(M_{3}\right)_{\varphi_{3}(k)} R_{j_{1}, j_{2}}^{(i)}
$$

As in Subsection 2.3 the player $P_{k}$ verifies the commitments of $P_{j}$ using usual pair-wise check for each $V S S(i)$.

\subsection{Additional Check on the Degree Reduction Phase}

Now we need to ensure that the player $P_{j}$ re-shares the correct vectors $x_{j_{1}, j_{2}}^{(i)}$ and in particular their true part. Unfortunately we can not apply directly the additional check procedure from step 4 . in the re-share protocol, because in the degree reduction phase we use two different access structures.

Let us choose the indices $j_{1} \in \varphi_{1}(j), j_{2} \in \varphi_{2}(j), k_{1} \in \varphi_{1}(k), k_{2} \in \varphi_{2}(k)$, $k_{3} \in \varphi_{3}(k)$ and $k_{4} \in \varphi_{4}(k)$. In order to perform this additional check we assume that there exist matrices $M_{3}$ and $M_{4}$, such that $M_{1} \diamond M_{2}=$ $M=M_{3} \diamond M_{4}$. This assumption means that we have $\left(M_{3}\right)_{k_{3}} \otimes\left(M_{4}\right)_{k_{4}}=$ $\left(M_{1}\right)_{k_{1}} \otimes\left(M_{2}\right)_{k_{2}}$ for some rows $k_{1}, k_{2}, k_{3}, k_{4}$ of the corresponding matrices. We first prove the following three equalities.

$$
\begin{aligned}
\left\langle y_{j_{1}, j_{2}, k_{3}}^{(i)}, \varepsilon_{3}^{T}\right\rangle & =\left\langle\left(M_{3}\right)_{k_{3}} R_{j_{1}, j_{2}}^{(i)}, \varepsilon_{3}^{T}\right\rangle \\
& =\left\langle\left(M_{3}\right)_{k_{3}},\left(R_{j_{1}, j_{2}}^{(i)}\right)_{1}\right\rangle=\left\langle\left(M_{3}\right)_{k_{3}}, x_{j_{1}, j_{2}}^{(i)}\right\rangle,
\end{aligned}
$$




$$
\begin{aligned}
\langle & \left.\left(M_{3}\right)_{k_{3}} \otimes\left(M_{4}\right)_{k_{4}}, x_{j_{1}, j_{2}}^{(i)} \otimes e_{i}\right\rangle=\left\langle\left(M_{3}\right)_{k_{3}}, x_{j_{1}, j_{2}}^{(i)}\right\rangle\left\langle\left(M_{4}\right)_{k_{4}}, e_{i}\right\rangle, \\
& \left\langle\left(M_{1}\right)_{k_{1}} \otimes\left(M_{2}\right)_{k_{2}}, v_{j_{1}} \otimes w_{j_{2}}\right\rangle=\left\langle\left(M_{1}\right)_{k_{1}}, v_{j_{1}}\right\rangle\left\langle\left(M_{2}\right)_{k_{2}}, w_{j_{2}}\right\rangle \\
= & \left(\left(M_{1}\right)_{k_{1}} v_{j_{1}}^{T}\right)\left(\left(M_{2}\right)_{k_{2}} w_{j_{2}}^{T}\right)=\left(v_{k_{1}}\left(M_{1}\right)_{j_{1}}^{T}\right)\left(w_{k_{2}}\left(M_{2}\right)_{j_{2}}^{T}\right) \\
= & \left\langle\left(M_{1}\right)_{j_{1}}, v_{k_{1}}\right\rangle\left\langle\left(M_{2}\right)_{j_{2}}, w_{k_{2}}\right\rangle .
\end{aligned}
$$

Now using (2) together with (3),(4), and (5) we are ready to prove that the player $P_{k}$ can make an additional check whether $P_{j}$ re-shared correctly the shares in the degree reduction phase. To perform this check $P_{k}$ uses his old shares $v_{k_{1}}$ and $w_{k_{2}}$ together with the newly received shares $y_{j_{1}, j_{2}, k_{3}}^{(i)}$ from $P_{j}$ and some public information.

$$
\left\langle\left(M_{1}\right)_{j_{1}}, v_{k_{1}}\right\rangle\left\langle\left(M_{2}\right)_{j_{2}}, w_{k_{2}}\right\rangle=\sum_{i=1}^{d_{4}}\left\langle\left(M_{4}\right)_{k_{4}}, e_{i}\right\rangle\left\langle y_{j_{1}, j_{2}, k_{3}}^{(i)}, \varepsilon_{3}^{T}\right\rangle .
$$

Note that we can simply choose $\mathcal{M}_{3}=\mathcal{M}_{1}$ and $\mathcal{M}_{4}=\mathcal{M}_{2}$, in this case we have $\Gamma_{1}=\Gamma_{3}$.

\subsection{The New Shares}

Finally, in order to complete the protocol we need to define the new shares. Recall that $j_{1} \in \varphi_{1}(j)$ and $j_{2} \in \varphi_{2}(j)$ if and only if $\left\{j_{1}, j_{2}\right\} \in \varphi(j)$. That is way we will denote $x_{j_{1}, j_{2}}^{(i)}$ and $y_{j_{1}, j_{2}, \varphi_{3}(k)}^{(i)}$ for $j_{1} \in \varphi_{1}(j)$ and $j_{2} \in \varphi_{2}(j)$ also by $x_{\varphi(j)}^{(i)}$ and by $y_{\varphi(j), \varphi_{3}(k)}^{(i)}$.

As we mentioned earlier in Section 3.4 for any group of players $\widetilde{G} \in \Gamma_{3}$ there exists a recombination vector $\widetilde{\lambda}_{\varphi_{3}(\widetilde{G})}$ such that they can reconstruct together the first coordinate of the vector $x_{\varphi(j)}^{(i)}$, i.e., $x_{\varphi(j)}^{(i)} \varepsilon_{3}$, for $i=$ $1, \ldots, d_{4}$ (reconstruction phase of $V S S(i)$ ) as follows:

$$
\begin{aligned}
\left(y_{\varphi(j), \varphi_{3}(\widetilde{G})}^{(i)} \varepsilon_{3}\right) \widetilde{\lambda}_{\varphi_{3}(\widetilde{G})}^{T} & =\left\langle\widetilde{\lambda}_{\varphi_{3}(\widetilde{G})}, y_{\varphi(j), \varphi_{3}(\widetilde{G})}^{(i)} \varepsilon_{3}\right\rangle \\
& =\sum_{k \in \widetilde{G}} \widetilde{\lambda}_{\varphi_{3}(k)}\left(y_{\varphi(j), \varphi_{3}(k)}^{(i)} \varepsilon_{3}\right)=x_{\varphi(j)}^{(i)} \varepsilon_{3},
\end{aligned}
$$

Note also that for any group of players $G \in \Gamma$ there exists a recombination vector $\lambda_{\varphi(G)}$ such that they can reconstruct together the product of the secrets $s_{1} s_{2}$.

$$
\begin{aligned}
\left(x_{\varphi(G)}^{(1)} \varepsilon_{3}\right) \lambda_{\varphi(G)}^{T} & =\left\langle\lambda_{\varphi(G)}, x_{\varphi(G)}^{(1)} \varepsilon_{3}\right\rangle \\
& =\left\langle\lambda_{\varphi(G)},\left(v_{\varphi_{1}(G)} \otimes w_{\varphi_{2}(G)}\right) \varepsilon\right\rangle=s_{1} s_{2} .
\end{aligned}
$$


(Here the last equality from Subsection 3.2 and the note from Subsection 3.3 are used.)

Now we are ready to define the new shares. Denote the list of good players by $\mathcal{L} \in \Gamma$, then $P_{k}$ computes his new shares as follows:

$$
z_{\varphi_{3}(k)}=\sum_{j \in \mathcal{L}} \lambda_{\varphi(j)} y_{\varphi(j), \varphi_{3}(k)}^{(1)} .
$$

For the new shares $z_{\varphi_{3}(k)}$ the pair-wise check holds:

$$
\begin{aligned}
\left(M_{3}\right)_{\varphi_{3}(i)} z_{\varphi_{3}(k)}^{T} & =\sum_{j \in \mathcal{L}} \lambda_{\varphi(j)}\left(M_{3}\right)_{\varphi_{3}(i)}\left(y_{\varphi(j), \varphi_{3}(k)}^{(1)}\right)^{T} \\
& =\left(\sum_{i \in \mathcal{L}} \lambda_{\varphi(j)} y_{\varphi(j), \varphi_{3}(i)}^{(1)}\right)\left(M_{3}\right)_{\varphi_{3}(k)}^{T}=z_{\varphi_{3}(i)}\left(M_{3}\right)_{\varphi_{3}(k)}^{T} .
\end{aligned}
$$

For any $\widetilde{G} \in \Gamma_{3}$ the players can reconstruct together the product $s_{1} s_{2}$ using (6) and (7) as follows:

$$
\begin{aligned}
\left(z_{\varphi_{3}(\widetilde{G})} \varepsilon_{3}\right) \widetilde{\lambda}_{\varphi_{3}(\widetilde{G})}^{T} & =\left\langle\widetilde{\lambda}_{\varphi_{3}(\widetilde{G})}, z_{\varphi_{3}(\widetilde{G})} \varepsilon_{3}\right\rangle=\sum_{k \in \widetilde{G}} \widetilde{\lambda}_{\varphi_{3}(k)}\left(z_{\varphi_{3}(k)} \varepsilon_{3}\right) \\
& =\sum_{k \in \widetilde{G}} \widetilde{\lambda}_{\varphi_{3}(k)}\left(\sum_{j \in \mathcal{L}} \lambda_{\varphi(j)}\left(y_{\varphi(j), \varphi_{3}(k)}^{(1)} \varepsilon_{3}\right)\right) \\
& =\sum_{j \in \mathcal{L}} \lambda_{\varphi(j)}\left(\sum_{k \in \widetilde{G}} \widetilde{\lambda}_{\varphi_{3}(k)}\left(y_{\varphi(j), \varphi_{3}(k)}^{(1)} \varepsilon_{3}\right)\right) \\
& =\sum_{j \in \mathcal{L}} \lambda_{\varphi(j)}\left(x_{\varphi(j)}^{(1)} \varepsilon_{3}\right)=s_{1} s_{2}
\end{aligned}
$$

At the end of the protocol each player $P_{k}$ possesses new shares $z_{\varphi_{3}(k)}$ of MSP $\mathcal{M}_{3}$ (computing the access structure $\Gamma_{3}$ ) of the product $s_{1} s_{2}$.

Lemma 1. Suppose that for the MSPs $\mathcal{M}_{1}$ and $\mathcal{M}_{2}$ there exist MSPs $\mathcal{M}_{3}$ and $\mathcal{M}_{4}$ such that

$$
\mathcal{M}_{1} \diamond \mathcal{M}_{2}=\mathcal{M}=\mathcal{M}_{3} \diamond \mathcal{M}_{4} .
$$

Let $\Gamma$ be the access structure computed by the strongly multiplicative resulting $M S P \mathcal{M}$ from $M S P s \mathcal{M}_{1}$ and $\mathcal{M}_{2}$ and/or from $M S P s \mathcal{M}_{3}$ and $\mathcal{M}_{4}$ and let also the access structures $\Gamma$ and $\Gamma_{i}$ for $i=1,2,3$ satisfy the conditions

$$
\Gamma_{A}^{\perp} \subseteq \Gamma \subseteq \Gamma_{1} \uplus \Gamma_{2}, \quad\left(\Gamma_{A} \uplus \Gamma_{A}\right)^{\perp} \subseteq \Gamma_{i} \text { for } i=1,2,3 .
$$

Then the "degree reduction" protocol is information-theoretically secure against $\left(\Delta_{1}, \Delta_{2}, \Delta_{A}\right)$-adversary. 
Due to lack of space we will not give a formal security proof for our protocol. However, to have a feeling why it is secure, note first that in the re-sharing phase every player could verify whether the "true" part of his share is correct or not. Then, as in the protocol from [4], the shares of the players (in our case the "true" part of the shares) have to satisfy a fixed linear relation, which allow every player to complain against incorrect re-sharing.

\subsection{Complexity Issues}

In this subsection we will follow [4]. Define $m s p_{\mathbb{F}}(f)$ to be the size of the smallest MSP over $\mathbb{F}$ computing a monotone boolean function $f$. Next define $\mu_{\mathbb{F}}(f)$ to be the size of the smallest multiplicative MSP over $\mathbb{F}$ computing $f$. Similarly, $\mu_{\mathbb{F}}^{*}(f)$ to be the size of the smallest strongly multiplicative MSP. In other words for a given adversary $A$ with adversary structure $\Delta_{A}$ we require for every set $B \in \Delta_{A}$ to have $B \notin \Gamma$, but $B^{c} \in \Gamma$. By definition, we have $m s p_{\mathbb{F}}(f) \leq \mu_{\mathbb{F}}(f) \leq \mu_{\mathbb{F}}^{*}(f)$. In [4] Cramer et al. characterized the functions that (strongly) multiplicative MSP's can compute, and proved that the multiplication property for an MSP can be assumed without loss of efficiency. In particular, for the passive (multiplicative) case they proved that $\mu_{\mathbb{F}}(f) \leq 2 m s p_{\mathbb{F}}(f)$ provided that $f$ is $Q^{2}$ function. Unfortunately there is no similar result for the strongly multiplicative case. Instead the authors in [4] proved that for an active adversary $\mu_{\mathbb{F}}^{*}(f)$ is bounded by the so-called "formula complexity".

In the recent paper of Nikov et al. [17] a different approach is considered. Recall that in that model given an $Q^{3}$ adversary $A$ we are looking for two access structures (resp. monotone boolean functions) $\Gamma_{1}$ and $\Gamma_{2}$ (resp. $f_{1}$ and $f_{2}$ ) such that their strongly multiplicative resulting MSP computes $\Gamma$ (resp. $f$ ). Or in other words for a given adversary $A$ with adversary structure $\Delta_{A}$ we require that for every set $B \in \Delta_{A}$ to have $B \notin \Gamma_{1}$, $B \notin \Gamma_{2}$ but $B^{c} \in \Gamma$. Let us define $\nu_{\mathbb{F}}(f)$ to be the size of the smallest strongly multiplicative resulting MSP over $\mathbb{F}$ computing $f$. How these two measures $\mu_{\mathbb{F}}^{*}(f)$ and $\nu_{\mathbb{F}}(f)$ are related as well as whether this new notion give us better measure for the complexity of an MPC is subject of ongoing research.

Acknowledgements. The authors would like to thank Ronald Cramer for the careful reading of earlier versions of the paper and for his constructive comments and remarks.

\section{References}

1. M. Ben-Or, S. Goldwasser and A. Wigderson, Completeness Theorems for Non- 
Cryptographic Fault-Tolerant Distributed Computation, Proc. ACM STOC'88, 1988, pp. 1-10.

2. D. Chaum, C. Crepeau and I. Damgard, Multi-Party Unconditionally Secure Protocols, Proc. ACM STOC'88, 1988, pp. 11-19.

3. R. Cramer, Introduction to Secure Computation, Lectures on Data Security - Modern Cryptology in Theory and Practice, Springer-Verlag LNCS 1561, 1999, pp. 1662 .

4. R. Cramer, I. Damgard and U. Maurer, General Secure Multi-Party Computation from any Linear Secret Sharing Scheme, Proc. EUROCRYPT 2000, SpringerVerlag LNCS 1807, 2000, pp. 316-334.

5. R. Cramer, S. Fehr, Optimal Black-Box Secret Sharing over Arbitrary Abelian Groups, Proc. CRYPTO 2002, Springer-Verlag LNCS 2442, 2002, pp. 272-287.

6. B. Chor, S. Goldwasser, S. Micali and B. Awerbuch, Verifiable Secret Sharing and Achieving Simultaneity in the Presence of Faults, Proc. of the IEEE 26th Annual Symp. on Foundations of Computer Science, 1985, pp. 383-395.

7. I.Damgard, An Error in the Mixed Adversary Protocol by Fitzi, Hirt and Maurer, Bricks Report, RS-99-2, 1999.

8. S. Fehr, U. Maurer, Linear VSS and Distributed Commitments Based on Secret Sharing and Pairwise Checks, Proc. CRYPTO 2002, Springer Verlag LNCS 2442, 2002, pp. 565-580.

9. M.Fitzi, M.Hirt and U.Maurer, Trading Correctness for Privacy in Unconditional Multi-Party Computation, Proc. CRYPT0'98, Springer-Verlag, LNCS 1462, 1998, pp. 121-136.

10. R. Gennaro, M. Rabin, T. Rabin, Simplified VSS and Fast-Track Multi-party Computations with Applications to Threshold Cryptography, Proc. ACM PODC'98, 1998.

11. O. Goldreich, S. Micali and A. Wigderson, How to Play Any Mental Game or a Completeness Theorem for Protocols with Honest Majority, Proc. ACM STOC'87, 1987, pp. 218-229.

12. M. Hirt, U. Maurer, Player Simulation and General Adversary Structures in Perfect Multi-party Computation, J. of Cryptology 13, 2000, pp. 31-60.

13. M. Karchmer, A. Wigderson, On Span Programs, Proc. of 8-th Annual Structure in Complexity Theory Conference, San Diego, California, 18-21 May 1993. IEEE Computer Society Press, pp. 102-111.

14. U. Maurer, Secure Multi-Party Computation Made Simple, 3rd Conference on Security in Communication Networks, September 12-13, 2002, Amalfi, Italy, SpringerVerlag LNCS 2576, 2003, pp. 14-28.

15. V. Nikov, S. Nikova, B. Preneel, J. Vandewalle, Applying General Access Structure to Proactive Secret Sharing Schemes, Proc. of the 23rd Symposium on Information Theory in the Benelux, May 29-31, 2002, Universite Catolique de Lovain (UCL), Lovain-la-Neuve, Belgium, pp. 197-206, Cryptology ePrint Archive: Report $2002 / 141$.

16. V. Nikov, S. Nikova, B. Preneel, J. Vandewalle, On Distributed Key Distribution Centers and Unconditionally Secure Proactive Verifiable Secret Sharing Schemes based on General Access Structure, INDOCRYPT 2002, Springer-Verlag LNCS 2551, 2002, pp. 422-437.

17. V. Nikov, S. Nikova, B. Preneel, Multi-Party Computation from any Linear Secret Sharing Scheme Secure against Adaptive Adversary: The Zero-Error Case, Cryptology ePrint Archive: Report 2003/006.

18. A. Shamir, How to share a secret, Commun. ACM 22, 1979, pp. 612-613. 